\title{
The early-type close binary CV Velorum revisited ${ }^{\star}$
}

\author{
K. Yakut ${ }^{1,2}$, C. Aerts ${ }^{1,3}$, and T. Morel ${ }^{1, \star \star}$ \\ 1 Instituut voor Sterrenkunde, Katholieke Universiteit Leuven, Celestijnenlaan 200 B, 3001 Leuven, Belgium \\ e-mail: conny@ster.kuleuven.be \\ 2 Department of Astronomy and Space Sciences, Ege University, 35100 İzmir, Turkey \\ 3 Department of Astrophysics, University of Nijmegen, PO Box 9010, 6500 GL Nijmegen, The Netherlands
}

Received 26 April 2006 / Accepted 25 January 2007

\section{ABSTRACT}

\begin{abstract}
Aims. Our goal was to improve the fundamental parameters of the massive close double-lined eclipsing B2.5V+B2.5V binary CV Velorum.

Methods. We gathered new high-resolution échelle spectroscopy on 13 almost consecutive nights covering two orbits. We computed a simultaneous solution to all the available high-quality radial-velocity and light data with the latest version of the Wilson-Deviney code.

Results. We obtained the following values for the physical parameters: $M_{1}=6.066(74) M_{\odot}, M_{2}=5.972(70) M_{\odot}, R_{1}=4.126(24) R_{\odot}$, $R_{2}=3.908(27) R_{\odot}, \log L_{1}=3.20(5) L_{\odot}$, and $\log L_{2}=3.14(5) L_{\odot}$. The quoted errors contain a realistic estimate of systematic uncertainties mainly stemming from the effective temperature estimation. We derived abundances for both components and found them to be compatible with those of B stars in the solar neighbourhood. We discovered low-amplitude periodic line-profile variations with the orbital frequency for both components. Their interpretation requires new data with a longer time span. The primary rotates subsynchronously while the secondary's $v \sin i$ and radius are compatible with synchronous rotation. We provide an update of the empirical mass-luminosity relation for main-sequence B stars which can be used for statistical predictions of masses or luminosities.
\end{abstract}

Key words. stars: early-type - stars: oscillations - stars: binaries: eclipsing - stars: fundamental parameters - stars: evolution - stars: individual: CV Velorum

\section{Introduction}

Calibrating stellar evolution models requires us to know the physical parameters of stars with a very high accuracy. Eclipsing and spectroscopic binaries with detached components allow us to obtain the required precise parameters. In this respect, $\mathrm{CV}$ Vel $\left(\mathrm{HD} 77464, V_{\mathrm{mag}}=6.7\right.$, spectral type $\left.\mathrm{B} 2.5 \mathrm{~V}+\mathrm{B} 2.5 \mathrm{~V}\right)$ and $\mathrm{a}$ few other massive binary stars have an important place in astrophysics. The star CV Vel is a well-known double-lined earlytype eclipsing binary system.

The binarity of CV Vel was originally established by van Houten (1950). The light curve of the system was first obtained by Gaposchkin (1955) photographically, leading to the first estimate of the physical parameters of the components. The system was studied spectroscopically by Feast (1954) and Andersen (1975). Feast (1954) obtained 34 spectra and derived the first spectroscopic orbital parameters of the system. He reported the spectral type of the components as $\mathrm{B} 2 \mathrm{~V}+\mathrm{B} 2 \mathrm{~V}$. Andersen (1975) performed a spectral study of the system based on data spread over 83 days and obtained much more accurate orbital parameters. Clausen \& Grønbech (1977) subsequently obtained a high quality light curve of CV Vel in the Strömgren bandpasses. These authors derived the orbital and physical parameters of the system, adding the radial velocities of Andersen (1975) to their data. The orbital period of the star is 6.889494(8) days.

$\mathrm{CV} \mathrm{Vel} \mathrm{is} \mathrm{an} \mathrm{important} \mathrm{massive} \mathrm{binary} \mathrm{whose} \mathrm{parameters} \mathrm{can}$ in principle be improved with modern high-quality data, which

\footnotetext{
* Based on spectroscopic observations gathered with the CORALIE spectrograph mounted on the $1.2 \mathrm{~m}$ Euler telescope at La Silla, Chile.

$\star \star$ External postdoctoral fellow of the European Space Agency.
}

is why we revisited this star after 30 years. We present a new detailed analysis of $\mathrm{CV}$ Vel considering all the available archival data to which we add new high-quality échelle spectra covering two consecutive weeks. We also search for a signature of oscillations by carefully analysing the line profiles of the components, given that they reside in the instability strip of the slowly pulsating B stars.

\section{New spectroscopic observations}

New spectra of CV Vel were obtained using the Swiss 1.2-m Euler telescope at La Silla, Chile and the CORALIE spectrograph. The system was observed on 13 almost successive nights in December 2001 and January 2002. Table 1 lists the spectral lines used in our radial-velocity analysis. The resolution of the spectra is 50000 and the integration time was in each case one hour to achieve an average $S / N$ ratio near 200 in the blue part of the spectrum.

The CORALIE spectra were reduced using the standard reduction pipeline (Baranne et al. 1996). For the study of the orbital motion, Gaussian fits to the absorption lines were made in order to derive their central wavelengths, thus transforming into the radial velocities for each of the considered lines. To illustrate the data quality, we show in Fig. 1 a selection of four lines for the two orbital phases with the largest line separation. It is clear from this figure that the primary has narrower lines than the secondary while being of similar temperature. This means that one of them must be asynchronous if the radii are similar, as we show is the case below. We come back to this point in Sect. 5 .

We list in Table 2 the average value of the measured radial velocities obtained from the five least blended spectral lines, i.e. 

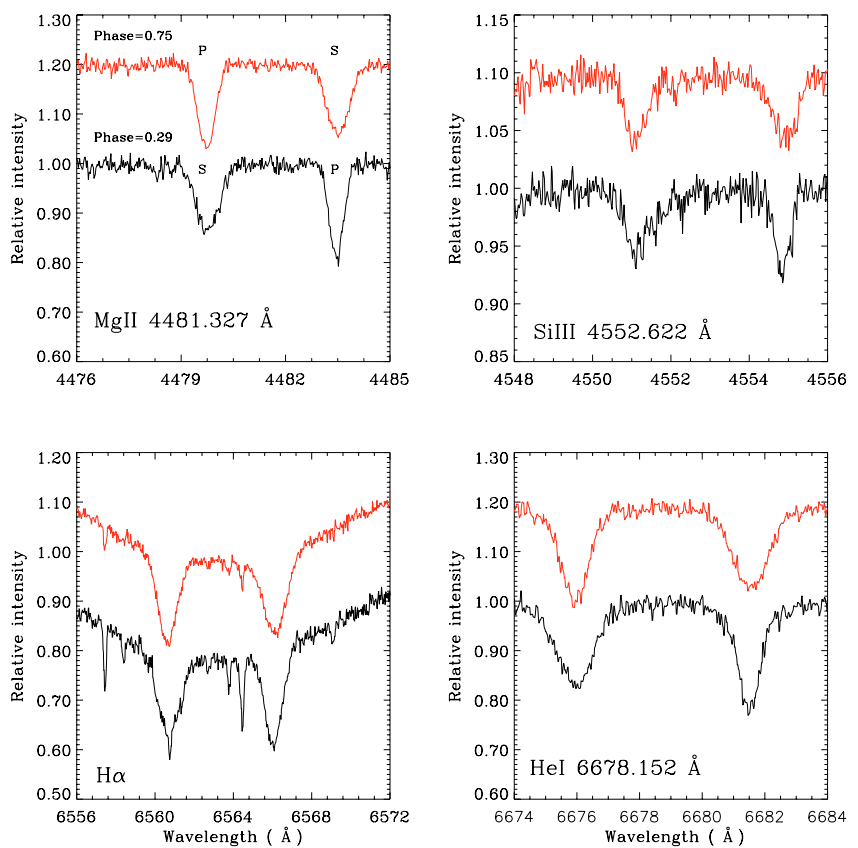

Fig. 1. Observed MgII 4481, SiIII 4552, H $\alpha$ and HeI 6678 line profiles at orbital phases 0.29 and 0.75 .

Table 1. Wavelength ranges and the spectral lines considered in our study.

\begin{tabular}{lll}
\hline \hline$\lambda_{\text {start }}$ & $\lambda_{\text {end }}$ & Considered lines \\
\hline 4102 & 4148 & HeI $\lambda 4121$, HeI $\lambda 4126$, SiII $\lambda 4128$, SiII $\lambda 4131$, \\
& & HeI $\lambda 4144$ \\
4464 & 4514 & HeI $\lambda 4471$, MgII $\lambda 4481$ \\
4530 & 4581 & SiIII $\lambda 4553$, SiIII $\lambda 4568$, SiIII $\lambda 4575$ \\
6528 & 6600 & H $\alpha$ \\
6671 & 6745 & HeI $\lambda 6678$ \\
\hline
\end{tabular}

He I $4120.993 \AA$, Mg II $4481.327 \AA$, Si III $4552.622 \AA$, Si III $4567.840 \AA$ and He I $6678.149 \AA$ lines. For each of these lines, the orbit was solved separately and the obtained results are summarized in Table 3. The internal precision of a single observation amounts to $\sim 1.0 \mathrm{~km} \mathrm{~s}^{-1}$ for the primary and $\sim 2.0 \mathrm{~km} \mathrm{~s}^{-1}$ for the secondary. We note a quite significant deviation among the $V_{0}$ values derived from the different lines. This is not uncommon in this part of the HR diagram (e.g. Mathias et al. 1994, for a discussion). It is interpreted in various ways, among which unknown blends within the lines, an asymmetric Stark effect among different lines (Struve \& Zebergs 1960) or the presence of a so-called Van Hoof effect (Van Hoof 1975) in pulsating stars. The latter effect is due to the fact that spectral lines form in different line forming regions in the stellar atmosphere, such that a running wave nature of the oscillations implies a shift in phase during the velocity cycle. Such an effect has clearly been detected in the pulsating B stars BW Vulpeculae and $\alpha$ Lupi (Mathias \& Gillet 1993). Our data have an insufficient time base and temporal resolution to test the presence of a Van Hoof effect in CV Vel (see also Sect. 6). Even if a Van Hoof effect is present, it would not explain the difference in $V_{0}$ for the two $\mathrm{Si}$ lines of the same triplet (SiIII $\lambda 4553$ and SiIII $\lambda 4568$ ). A similar unexplained average velocity shift between the same lines in this Si triplet was encountered by De Cat et al. (2000) in their analysis of several hundred line-profile measurements, of very high $\mathrm{S} / \mathrm{N}$, of the B0.5III star $\varepsilon$ Per. As there, we are unable to offer a good interpretation of this shift. We will take it into account
Table 2. Radial velocity values for CV Vel computed in this study.

\begin{tabular}{lrrrrr}
\hline \hline HJD $(2452000+)$ & Phase & $V_{1}$ & $\mathrm{O}-\mathrm{C}_{1}$ & $V_{2}$ & ${\mathrm{O}-\mathrm{C}_{2}}$ \\
\hline 272.6910 & 0.000 & & & 20.1 & -3.7 \\
272.8130 & 0.017 & -2.5 & -8.0 & 39.0 & 0.9 \\
273.6340 & 0.137 & -76.9 & -1.4 & 123.6 & 3.1 \\
273.8430 & 0.167 & -87.2 & 2.0 & 135.2 & 0.9 \\
274.6680 & 0.287 & -100.2 & 0.8 & 149.8 & 3.6 \\
274.8110 & 0.308 & -95.4 & 0.5 & 142.3 & 1.2 \\
276.6810 & 0.579 & 83.0 & -0.9 & -39.1 & 2.4 \\
276.7990 & 0.596 & 95.8 & 0.4 & -52.9 & 0.3 \\
277.5890 & 0.711 & 145.5 & 0.6 & -103.1 & 0.4 \\
277.7320 & 0.731 & 148.4 & 0.7 & -107.9 & -1.7 \\
278.5650 & 0.852 & 120.5 & -1.1 & -78.0 & 1.8 \\
278.7740 & 0.883 & 104.8 & -0.3 & -65.4 & -2.5 \\
279.5940 & 0.002 & & & $17.5 ?$ & -7.9 \\
279.7250 & 0.021 & 0.9 & -2.0 & 39.2 & -1.5 \\
279.8420 & 0.038 & -8.4 & 1.9 & 54.0 & -0.1 \\
280.5550 & 0.141 & -78.9 & -1.1 & 122.6 & -0.2 \\
280.6970 & 0.162 & -87.2 & -0.1 & 132.8 & 0.6 \\
280.7620 & 0.171 & -88.9 & 1.9 & 136.2 & 0.3 \\
281.5540 & 0.286 & -100.7 & 0.4 & 146.3 & 0.0 \\
281.6820 & 0.305 & -96.6 & 0.1 & 140.0 & -1.9 \\
281.7840 & 0.320 & -91.5 & 0.5 & 134.4 & -2.7 \\
282.5610 & 0.433 & -26.7 & 1.6 & 73.7 & 1.2 \\
282.6970 & 0.452 & -12.8 & 0.9 & 59.3 & 1.7 \\
283.5830 & 0.581 & 85.1 & -0.1 & -46.8 & -4.0 \\
283.6840 & 0.596 & 95.3 & 0.3 & -54.4 & -1.6 \\
283.8000 & 0.612 & 105.9 & 0.4 & -62.9 & 0.5 \\
284.5480 & 0.721 & 147.3 & 0.8 & -103.6 & 1.5 \\
284.6990 & 0.743 & 148.8 & 0.6 & -103.2 & 3.6 \\
284.7720 & 0.753 & 149.0 & 0.8 & -103.0 & 3.8 \\
285.6550 & 0.882 & 107.4 & 1.6 & -65.7 & -2.0 \\
\hline & & & & &
\end{tabular}

as a systematic uncertainty in the derivation of the orbital and fundamental parameters of the star (see below). Although the eccentricity, $e$, was first regarded as a free parameter, its value always appeared to be zero, within the error range, so we do not list it in Table 3.

The fifth column of Table 4 gives the orbital solution from the averaged radial velocities of our study. The average radial velocity values are plotted versus the orbital phase for each of the components in Fig. 2. The phases were calculated using the ephemeris given by Clausen \& Grønbech (1977):

Pri. Min. = HJD $2442048.66894(14)+6.889494(8) \times E$.

\section{Simultaneous radial-velocity and light curve analysis}

To measure the $\sum \omega(\mathrm{O}-\mathrm{C})^{2}$ values, given in Table 4 , we reanalyzed the data of Feast (1954) and Andersen (1975). The data of Andersen (1975) and those of our paper are of similar quality while those of Feast (1954) are of lower quality, as emphasised by Andersen (1975). For this reason, we omitted Feast's data in the remainder of this paper.

We combined the newly obtained radial-velocity values (Table 2) with those previously obtained by Anderson (1975). This gave a total of 62 radial-velocity values for each of the components, to which we assigned equal weights. As can be seen in Table 4, we find an offset in average velocity $V_{0}$ between the two radial-velocity sets. This could be due to the difference in spectral lines used in the two studies, but it may also be that we find a downward trend in $V_{0}$ over time due to the presence of a third body. We have too few epochs to model any $V_{0}$ trend for the time 
Table 3. Spectroscopic orbital elements of CV Vel for different spectral lines. See text for details. The formal standard errors $\sigma$ are given in parentheses in the last digit quoted.

\begin{tabular}{|c|c|c|c|c|c|c|c|c|}
\hline Ion & $\begin{array}{l}\text { Lines } \\
\AA\end{array}$ & $\begin{array}{l}K_{1} \\
\mathrm{~km} \mathrm{~s}^{-1}\end{array}$ & $\begin{array}{l}K_{2} \\
\mathrm{~km} \mathrm{~s}^{-1}\end{array}$ & $\begin{array}{l}m_{1} \sin ^{3} i \\
M_{\odot}\end{array}$ & $\begin{array}{l}m_{2} \sin ^{3} i \\
M_{\odot}\end{array}$ & $\begin{array}{l}a_{1} \sin i \\
R_{\odot}\end{array}$ & $\begin{array}{l}a_{2} \sin i \\
R_{\odot}\end{array}$ & $\begin{array}{l}V_{0} \\
\mathrm{~km} \mathrm{~s}^{-1}\end{array}$ \\
\hline $\mathrm{He} \mathrm{I}$ & 4120.993 & $126.8(5)$ & $128.6(5)$ & 5.988 & 5.904 & 17.261 & 17.545 & $16.2(2)$ \\
\hline Mg II & 4481.327 & $126.9(4)$ & $128.6(4)$ & 5.993 & 5.913 & 17.290 & 17.528 & $18.2(2)$ \\
\hline Si III & 4552.622 & $125.2(6)$ & $128.2(6)$ & 5.876 & 5.739 & 17.037 & 17.447 & $23.5(3)$ \\
\hline Si III & 4567.840 & $127.0(5)$ & $128.2(5)$ & 5.960 & 5.904 & 17.315 & 17.473 & $24.7(3)$ \\
\hline $\mathrm{He} \mathrm{I}$ & 6678.149 & $127.0(4)$ & $128.6(4)$ & 5.997 & 5.923 & 17.287 & 17.498 & $24.2(2)$ \\
\hline
\end{tabular}

Table 4. Spectroscopic orbital parameters of CV Vel. The standard errors $\sigma$ are given in parentheses in the last digit quoted.

\begin{tabular}{llllll}
\hline \hline Parameter & Unit & Feast (1954) & Andersen (1975) & This paper & This paper + Andersen (1975) \\
\hline$K_{1}$ & $\mathrm{~km} \mathrm{~s}^{-1}$ & $122.2(6)$ & $127.0(3)$ & $126.5(3)$ & $127.0(2)$ \\
$K_{2}$ & $\mathrm{~km} \mathrm{~s}^{-1}$ & $126.6(6)$ & $129.2(4)$ & $128.6(3)$ & $129.1(2)$ \\
$V_{0}$ & $\mathrm{~km} \mathrm{~s}^{-1}$ & $27.9(1.1)$ & $24.3(5)$ & $21.7(2)$ & $21.7(1)$ \\
$q$ & $m_{2} / m_{1}$ & 0.965 & 0.983 & 0.984 & 0.984 \\
$a \sin i$ & $R_{\odot}$ & 33.72 & 34.87 & 34.722 & 34.857 \\
$m_{1} \sin ^{3} i$ & $M_{\odot}$ & 5.61 & 6.07 & 5.974 & 6.021 \\
$m_{2} \sin ^{3} i$ & $M_{\odot}$ & 5.41 & 5.96 & 5.876 & 5.927 \\
$\sum(\mathrm{O}-\mathrm{C})^{2}$ & & 0.08 & 0.01 & 0.01 & 0.01 \\
\hline
\end{tabular}

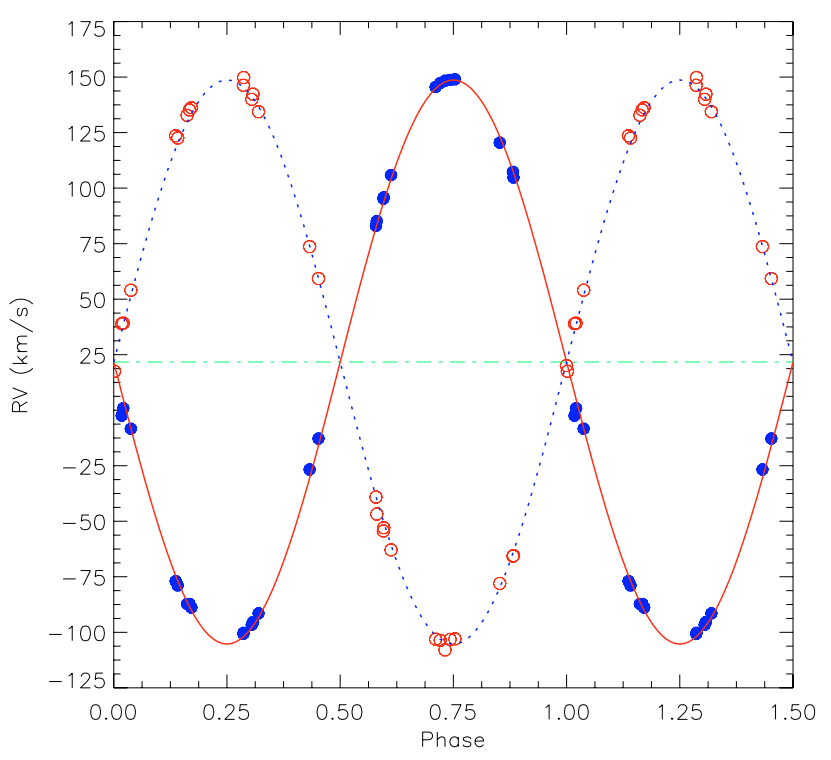

Fig. 2. The radial velocity observations of CV Vel versus the orbital phase. The filled and open circles correspond to the velocities of the primary and the secondary, respectively. The data are listed in Table 2 and the sine curve corresponds to the elements given in Table 4 . The center-of-mass velocity is indicated by the dashed-dotted line.

being. For this reason, we shifted the data towards the CORALIE $V_{0}$ value, keeping in mind that this systematic uncertainty adds to the uncertainty of the overall solution.

These 62 radial-velocity values were then combined with the light curves obtained in the Strömgren ( $u b v y)$ filters by Clausen \& Grønbech (1977), kindly made available to us by these authors. The $u$ band data show a somewhat larger scatter than the other filters so we used different weights. We determined the weights for the $u v b y$ bands from the observing errors given by Clausen \& Grønbech (1977), resulting in the values 1.7, 2.5, 2.0, and 2.0 for $u, v, b$ and $y$, respectively.

Previous light curve analyses of the system were performed using the WINK method by Clausen \& Grønbech (1977) (second column of Table 5). Clausen \& Grønbech (1977) used a linear limb-darkening law, fixing the temperature of the primary star at $18300 \mathrm{~K}$. They determined solutions for each colour, assuming different values for $k=r_{2} / r_{1}$ (see their Table 4). In this work, the light and radial-velocity curves were analyzed simultaneously using the 2005 September version of the Wilson-Deviney code (hereafter WD, Wilson \& Devinney 1971; Wilson 1994). Mode 2 of the WD code, which assumes that both of the components are detached, was adopted. In the final solution, we fixed the following parameters: the limb-darkening coefficients (taken from Diaz-Cordoves \& Gimenez 1992, see Table 5), the values of the gravity darkening coefficients and the albedos. The adjustable parameters are the inclination $i$, the temperature of the secondary component $T_{2}$, the luminosities $L_{1 b}$ and $L_{1 y}$, and the surface potentials $\Omega_{1}$ and $\Omega_{2}$. The differential correction (DC) code was run until the corrections to the input parameters were lower than their errors.

To obtain a simultaneous solution, different possibilities were tested. We varied the primary's temperature in the range $17800-19000 \mathrm{~K}$ with $50 \mathrm{~K}$ steps and found that $T_{1}=18000 \mathrm{~K}$ gave the best solution with the lowest formal errors for the unknown quantities and the lowest $\sum(\mathrm{O}-\mathrm{C})^{2}$. However, the solutions with $T_{1}$ between $18000 \mathrm{~K}$ and $19000 \mathrm{~K}$ were almost indistinghuishable. This is in agreement with the solution proposed by Clausen \& Grønbech (1977), who assigned $T_{1}=18200 \mathrm{~K}$ to the mean component of the system and considered an uncertainty of $500 \mathrm{~K}$. In selecting the best solution, we first assumed the system to be eccentric leaving the $e$ and $\omega$ values free. As a result we always obtained $e \leq 0.001$. The final results are given in Table 5 for each band separately, as well as for the simultaneous solution including all photometric data. We find a similar orbital inclination value as Clausen \& Grønbech (1977). The computed light and radial-velocity curves for the parameters given in Table 5 are shown by the solid lines, and are compared with all the observations in Fig. 3.

\section{Abundance determination}

Our spectra are of sufficient quality, and cover a wide enough wavelength range, to undertake an abundance analysis for the 
Table 5. Orbital parameters and photometric elements, with their formal errors, of CV Vel.

\begin{tabular}{|c|c|c|c|c|c|c|}
\hline Parameter & $\begin{array}{l}\text { CG } \\
\text { Value }(\sigma)\end{array}$ & $\begin{array}{l}u \\
\text { Value }(\sigma)\end{array}$ & $\begin{array}{l}v \\
\text { Value }(\sigma)\end{array}$ & $\begin{array}{l}b \\
\text { Value }(\sigma)\end{array}$ & $\begin{array}{l}y \\
\text { Value }(\sigma)\end{array}$ & $\begin{array}{l}\text { ubvy } \\
\text { Value }(\sigma)\end{array}$ \\
\hline \multicolumn{7}{|l|}{ Geometric parameters: } \\
\hline$i\left(^{\circ}\right)$ & $86.59(5)$ & $86.66(2)$ & $86.62(2)$ & $86.62(2)$ & $86.62(2)$ & $86.63(2)$ \\
\hline$\Omega_{1}$ & - & $9.500(9)$ & $9.445(9)$ & $9.451(9)$ & $9.445(9)$ & $9.456(10)$ \\
\hline$\Omega_{2}$ & - & $9.852(11)$ & $9.807(11)$ & $9.805(11)$ & 9.794(11) & $9.804(12)$ \\
\hline$a$ & - & $34.897(19)$ & $34.896(19)$ & $34.891(19)$ & $34.898(19)$ & $34.899(20)$ \\
\hline$q$ & - & $0.9837(9)$ & $0.9842(9)$ & $0.9845(9)$ & $0.9838(9)$ & $0.9835(10)$ \\
\hline \multicolumn{7}{|l|}{ Fractional radii of the primary } \\
\hline$r_{1 \text { pole }}$ & - & $0.1172(4)$ & $0.1180(3)$ & $0.1181(3)$ & $0.1182(3)$ & $0.1172(1)$ \\
\hline$r_{1 \text { point }}$ & - & $0.1177(4)$ & $0.1186(3)$ & $0.1186(3)$ & $0.1187(3)$ & $0.1184(1)$ \\
\hline$r_{1 \text { side }}$ & - & $0.1174(4)$ & $0.1182(3)$ & $0.1183(3)$ & $0.1184(3)$ & $0.1181(1)$ \\
\hline$r_{1 \text { back }}$ & - & $0.1176(4)$ & $0.1185(3)$ & $0.1186(3)$ & $0.1186(3)$ & $0.1184(1)$ \\
\hline$r_{1 \text { mean }}$ & $0.117(1)$ & $0.1175(4)$ & $0.1183(3)$ & $0.1184(3)$ & $0.1185(3)$ & $0.1182(1)$ \\
\hline \multicolumn{7}{|l|}{ Fractional radii of the secondary } \\
\hline$r_{2}$ pole & - & $0.1113(4)$ & $0.1117(3)$ & $0.1118(3)$ & $0.1118(3)$ & $0.1117(2)$ \\
\hline$r_{2}$ point & - & $0.1117(4)$ & $0.1122(3)$ & $0.1122(3)$ & $0.1123(3)$ & $0.1121(2)$ \\
\hline$r_{2}$ side & - & $0.1115(4)$ & $0.1119(3)$ & $0.1119(3)$ & $0.1120(3)$ & $0.1119(2)$ \\
\hline$r_{2 \text { back }}$ & - & $0.1117(4)$ & $0.1121(3)$ & $0.1121(3)$ & $0.1122(3)$ & $0.1121(2)$ \\
\hline$r_{2 \text { mean }}$ & $0.113(1)$ & $0.1115(4)$ & $0.1120(3)$ & $0.1120(3)$ & $0.1121(3)$ & $0.1119(2)$ \\
\hline \multicolumn{7}{|l|}{ Radiative parameters: } \\
\hline$T_{1}{ }^{*}(\mathrm{~K})$ & 18200 & 18000 & 18000 & 18000 & 18000 & 18000 \\
\hline$T_{2}(\mathrm{~K})$ & 18060 & $17813(50)$ & $17703(50)$ & $17818(50)$ & $17815(50)$ & $17790(50)$ \\
\hline Albedo* $\left(A_{1}=A_{2}\right)$ & 1.0 & 1.0 & 1.0 & 1.0 & 1.0 & 1.0 \\
\hline \multicolumn{7}{|l|}{ Limb darkening coefficients*: } \\
\hline \multicolumn{7}{|l|}{ Square Law: } \\
\hline$x_{1 \mu}^{\text {bol }}$ & - & 0.054 & & & & 0.054 \\
\hline$x_{1 v}^{\text {bol }}$ & - & & -0.089 & & & -0.089 \\
\hline$x_{1 h}^{\text {bol }}$ & - & & & -0.078 & & -0.078 \\
\hline$x_{1 y}^{b b}$ & - & & & & -0.067 & -0.067 \\
\hline$x_{2 u}^{\text {bol }}$ & - & 0.059 & & & & 0.059 \\
\hline$x_{2}^{\text {bol }}$ & - & & -0.073 & & & -0.073 \\
\hline$x_{2 h}^{2 v}$ & - & & & -0.078 & & -0.078 \\
\hline$x_{2 u}^{2 b}$ & - & & & & -0.067 & -0.067 \\
\hline$y_{1 u}^{2 y}$ & - & 0.492 & & & & 0.492 \\
\hline$y_{1 v}^{\text {bol }}$ & - & & 0.718 & & & 0.718 \\
\hline$y_{1 b}^{\text {bol }}$ & - & & & 0.666 & & 0.666 \\
\hline$y_{1 y}^{\text {bol }}$ & - & & & & 0.581 & 0.581 \\
\hline$y_{2 u}^{\text {bol }}$ & - & 0.550 & & & & 0.550 \\
\hline$y_{2 v}^{\text {bol }}$ & - & & 0.611 & & & 0.611 \\
\hline$y_{2 b}^{\text {bol }}$ & - & & & 0.663 & & 0.663 \\
\hline $\begin{array}{l}y_{2 b} \\
y_{2 u}^{\text {bol }}\end{array}$ & - & & & & 0.583 & 0.583 \\
\hline Gravity brightening* $\left(g_{1}=g_{2}\right)$ & 1.0 & 1.0 & 1.0 & 1.0 & 1.0 & 1.0 \\
\hline \multicolumn{7}{|l|}{ Luminosity ratio } \\
\hline$\left(\frac{L_{1}}{L_{1}+L_{2}}\right)_{u}$ & $0.519(20)$ & $0.533(13)$ & & & & $0.534(13)$ \\
\hline$\left(\frac{L_{1}}{L_{1}+L_{2}}\right)_{v}$ & $0.517(20)$ & & $0.531(12)$ & & & $0.528(12)$ \\
\hline$\left(\frac{L_{1}}{L_{1}+L_{2}}\right)_{b}$ & $0.517(20)$ & & & $0.532(12)$ & & $0.532(12)$ \\
\hline$\left(\frac{L_{1}+L_{2}}{L_{1}+L_{2}}\right)_{y}$ & $0.516(20)$ & & & & $0.531(12)$ & $0.532(12)$ \\
\hline$\sum(\mathrm{O}-\mathrm{C})^{2}$ & - & 0.028 & 0.026 & 0.023 & 0.025 & 0.064 \\
\hline
\end{tabular}

CG: Clausen \& Grønbech (1977), the other columns represent results obtained in this work. The parameters with a superscript $*$ were kept fixed.

two components of the CV Vel system. The non-local thermodynamic equilibrium (NLTE) abundances of $\mathrm{He}, \mathrm{C}, \mathrm{N}, \mathrm{O}, \mathrm{Mg}$, $\mathrm{Al}, \mathrm{Si}, \mathrm{S}$ and $\mathrm{Fe}$ were calculated using the latest versions of the line formation codes DETAIL/SURFACE and plane-parallel, fully line-blanketed Kurucz atmospheric models (Kurucz 1993). Curve-of-growth techniques were used to determine the abundances using the equivalent widths $(E W \mathrm{~s})$ of a set of spectral lines measured on a mean spectrum created by averaging the five CORALIE spectra taken at $\phi \sim 0.73$ (see Morel et al. 2006, for the line list used). These $E W \mathrm{~s}$ were subsequently corrected to account for the dilution by the companion's continuum, assuming the luminosity ratio given in Table 7 . The two components have essentially the same effective temperature, so that these correcting factors can be considered independent of wavelength.

We first computed the abundances assuming the $\log g$ and $T_{\text {eff }}$ values obtained from the light curve analysis (Table 7), but found that the Si II/III ionization balance is not well fullfilled in that case. Instead, a slightly higher value of $T_{\text {eff }}=$ $19000 \pm 500 \mathrm{~K}$ is needed for both components. Because of this ambiguity surrounding the temperature determination, we have estimated the abundances using both the photometric and the spectroscopic values (hereafter $T_{\text {photo }}$ and $T_{\text {ionization, }}$, 


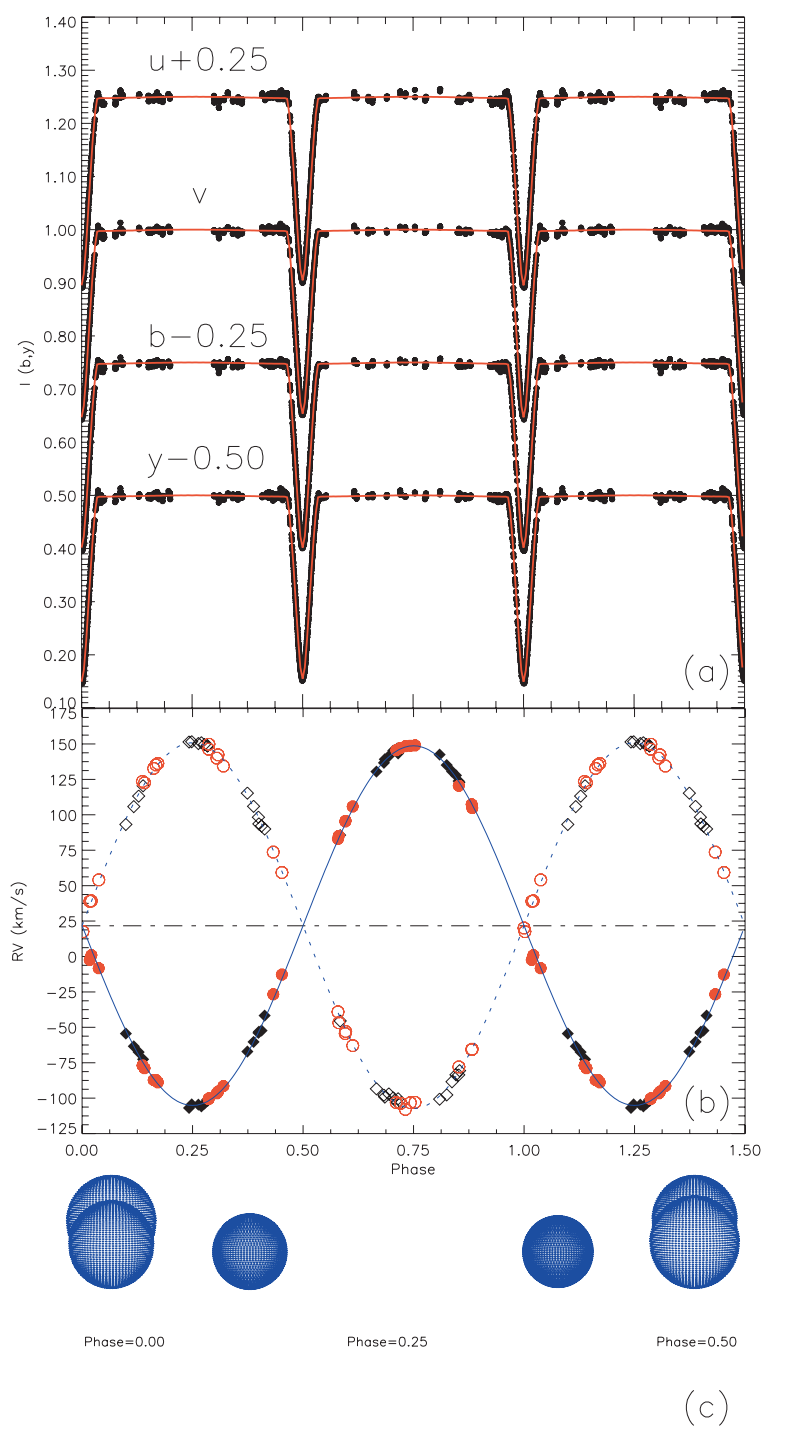

Fig. 3. The observed and computed a) light and b) radial-velocity curves of CV Vel. The curve in the $u, b$ and $y$ bands are moved by $+0.25,-0.25$ and -0.50 , respectively, in intensity for visibility. Circles and squares denote the data in this study and Anderson (1975) respectively. The center-of-mass velocity is indicated by the dashed-dotted line. c) 3D representation of CV Vel at phases $0.00,0.25$ and 0.50 .

respectively). The uncertainties on the elemental abundances were calculated by adding in quadrature the internal errors (i.e. the line-to-line scatter) and the errors arising from the uncertainty on the microturbulent velocity, $\xi$. The latter quantity is usually inferred in B stars by requiring no dependence between the abundances yielded by lines of an ionic species with strong features (e.g. O II) and their strength. However, most metallic lines are intrinsically weak in the relevant temperature range (measured $E W \mathrm{~s} \lesssim 20 \mathrm{~m} \AA$ ), such that it is virtually impossible to constrain the microturbulence. We adopt in the following a canonical value $\xi=5 \pm 5 \mathrm{~km} \mathrm{~s}^{-1}$, with the large conservative error bars encompassing all plausible values for B dwarfs. This translates into poorly defined abundances for the elements exhibiting only relatively strong lines, in particular $\mathrm{He}, \mathrm{Si}$ and $\mathrm{Mg}$.

The results for the two choices of $T_{\text {eff }}$ are provided in Table 6 . No abundances are found to deviate at more than the $3 \sigma$ level from the typical values found for early B dwarfs in the solar neighbourhood (Daflon \& Cunha 2004) for both $T_{\text {eff }}$ estimates. Therefore, there is no convincing evidence for departures from a scaled solar composition. The nominal abundance values are systematically higher in the secondary than in the primary. As the chemical composition of the two components is expected to be identical for such a young, detached system with no past or ongoing episodes of mass transfer, this strongly argues for a higher microturbulent velocity in the secondary. This difference is, however, not sufficient to explain the discrepant rotation rates of the two components (see Sect. 6 below).

Recent works point to the existence of a population of slowly-rotating B-type (magnetic) pulsators exhibiting an unexpected nitrogen excess at their surface, which could possibly be attributed to deep mixing or diffusion effects (Briquet \& Morel 2007; Morel et al. 2006). A similar phenomenon cannot be firmly established in either of the two components of CV Vel: while the ratio of the abundances of $\mathrm{N}$ and $\mathrm{C}([\mathrm{N} / \mathrm{C}])$ is comparable to the values previously reported for the $\mathrm{N}$-rich $\beta$ Cephei and slowly pulsating $B$ stars, the $[\mathrm{N} / \mathrm{O}]$ ratio is indistinguishable from solar. This conclusion holds irrespective of the temperature scale adopted.

Regarding the derivation of the physical parameters of the component stars, we are thus left with the conclusion that a second systematic uncertainty occurs, besides the one induced by the offset in $V_{0}$. We forced the effective temperature of the primary to $19000 \mathrm{~K}$ and checked the orbital solutions from WD discussed in the previous section. As already mentioned, the differences in the orbital parameter values are small and compatible with the uncertainties listed there. While deriving the physical parameters of the system in the next section, we propagated the errors caused by the systematic uncertainty in effective temperature in order to achieve realistic errors.

\section{Physical parameters of the system}

The physical parameters of CV Vel first obtained by Gaposchkin (1955) were $M_{1}=5.618 M_{\odot}, M_{2}=5.418 M_{\odot}, R_{1}=4.26 R_{\odot}$, and $R_{2}=4.16 R_{\odot}$. Andersen (1975) reported the parameters of the components as $M_{1,2}=6.05 M_{\odot}, R_{1,2}=4.05 R_{\odot}$, and $\log T_{\text {eff }}=$ 4.26. Clausen \& Grønbech (1977) subsequently obtained $M_{1}=6.10 M_{\odot}, M_{2}=5.99 M_{\odot}, R_{1}=4.10 R_{\odot}, R_{2}=3.95 R_{\odot}$, $T_{1}=18200 \mathrm{~K}$ and $T_{2}=18060 \mathrm{~K}$.

We summarize the obtained parameters of the system and of the components obtained from our simultaneous fit in Table 7 . In computing them, we took the values of the light curve estimates for the effective temperature of the components. We argue that the photometric data are of high quality while the spectroscopic temperature estimates suffer from the unknown microturbulence. However, we take into account a systematic uncertainty of $500 \mathrm{~K}$ for the temperature. This equals the uncertainty derived from the silicon ionisation balance and is a factor of ten higher than the formal error that resulted from our WD analysis, so we consider this to be a safe conservative approach. We propagated this uncertainty for all the derived quantities relying on the effective temperature.

We derived the component's bolometric corrections from the temperatures using the interpolation formula given by Balona (1994) for G5 to early-type stars. In order to find the distance of the system, we used the $E(B-V)$ value of 0.08 from Savage et al. (1985) resulting in $d=553 \mathrm{pc}$.

Our results are in very good agreement with those obtained by Clausen \& Grønbech (1977). We were unable to achieve more precise values than they did, due to the uncertainty on the effective temperature resulting from our spectroscopic analysis.

We compared our observational results for CV Vel with evolutionary models computed by De Cat et al. (2006) with the Code 
Table 6. Mean NLTE abundances of the primary and secondary components of CV Vel (on the scale in which $\log \epsilon[\mathrm{H}]=12$ ), along with the total $1-\sigma$ uncertainties. The number of used lines is given in brackets.

\begin{tabular}{lccccc}
\hline \hline & & \multicolumn{2}{c}{$T_{\text {eff }} \equiv T_{\text {photo }}=18000 \mathrm{~K}$} & \multicolumn{2}{c}{$T_{\text {eff }} \equiv T_{\text {ionization }}=19000 \mathrm{~K}$} \\
& OB stars & Primary & Secondary & Primary & Secondary \\
\hline $\mathrm{He} / \mathrm{H}$ & 0.085 & $0.053 \pm 0.029(6)$ & $0.097 \pm 0.033(6)$ & $0.040 \pm 0.022(6)$ & $0.073 \pm 0.031(6)$ \\
$\log \epsilon(\mathrm{C})$ & $\sim 8.2$ & $8.08 \pm 0.10(4)$ & $8.29 \pm 0.17(2)$ & $7.89 \pm 0.07(4)$ & $8.11 \pm 0.17(2)$ \\
$\log \epsilon(\mathrm{N})$ & $\sim 7.6$ & $7.93 \pm 0.13(7)$ & $8.16 \pm 0.16(6)$ & $7.69 \pm 0.13(7)$ & $7.92 \pm 0.17(6)$ \\
$\log \epsilon(\mathrm{O})$ & $\sim 8.5$ & $8.74 \pm 0.18(8)$ & $8.85 \pm 0.20(4)$ & $8.44 \pm 0.17(8)$ & $8.55 \pm 0.19(4)$ \\
$\log \epsilon(\mathrm{Mg})$ & $\sim 7.4$ & $7.01 \pm 0.40(1)$ & $7.26 \pm 0.45(1)$ & $7.13 \pm 0.40(1)$ & $7.39 \pm 0.39(1)$ \\
$\log \epsilon(\mathrm{Al})$ & $\sim 6.1$ & $6.32 \pm 0.18(3)$ & $6.48 \pm 0.17(3)$ & $6.12 \pm 0.18(3)$ & $6.27 \pm 0.17(3)$ \\
$\log \epsilon(\mathrm{Si})$ & $\sim 7.2$ & $6.97 \pm 0.44(7)$ & $7.15 \pm 0.61(7)$ & $6.93 \pm 0.28(7)$ & $7.11 \pm 0.44(7)$ \\
$\log \epsilon(\mathrm{S})$ & $\sim 7.2$ & $7.02 \pm 0.21(5)$ & $7.25 \pm 0.23(2)$ & $7.08 \pm 0.19(5)$ & $7.29 \pm 0.25(2)$ \\
$\log \epsilon(\mathrm{Fe})$ & $\sim 7.3^{a}$ & $7.20 \pm 0.20(3)$ & $7.46 \pm 0.18(2)$ & $7.05 \pm 0.15(3)$ & $7.34 \pm 0.23(2)$ \\
\hline$[\mathrm{N} / \mathrm{C}]$ & $\sim-0.6$ & $-0.15 \pm 0.17$ & $-0.13 \pm 0.24$ & $-0.20 \pm 0.15$ & $-0.19 \pm 0.25$ \\
{$[\mathrm{~N} / \mathrm{O}]$} & $\sim-0.9$ & $-0.81 \pm 0.23$ & $-0.69 \pm 0.26$ & $-0.75 \pm 0.22$ & $-0.63 \pm 0.26$ \\
\hline
\end{tabular}

${ }^{a}$ From Morel et al. (2006).

Table 7. Absolute parameters for CV Vel. The errors are given in parentheses and take into account the systematic uncertainties encountered for $V_{0}$ and for the effective temperature of the components.

\begin{tabular}{llll}
\hline \hline & Unit & Primary component & Secondary component \\
\hline Mass $(M)$ & $M_{\odot}$ & $6.066(74)$ & $5.972(70)$ \\
Radius $(R)$ & $R_{\odot}$ & $4.126(24)$ & $3.908(27)$ \\
Effective temperature $\left(\log T_{\text {eff }}\right)$ & $K$ & $4.255(12)$ & $4.250(12)$ \\
Luminosity $(\log L)$ & $L_{\odot}$ & $3.204(48)$ & $3.137(48)$ \\
Surface gravity $(\log g)$ & $\mathrm{cgs}$ & $3.99(6)$ & $4.03(6)$ \\
Bolometric correction $(\mathrm{BC})$ & $\mathrm{mag}$ & $-1.68(5)$ & $-1.66(5)$ \\
Absolute bolometric magnitude $\left(M_{\mathrm{bol}}\right)$ & $\mathrm{mag}$ & $-3.26(3)$ & $-3.09(3)$ \\
Absolute visual magnitude $\left(M_{\mathrm{V}}\right)$ & $\mathrm{mag}$ & $-1.58(6)$ & $-1.43(5)$ \\
Semi-major axis $(a)$ & $R_{\odot}$ & \multicolumn{2}{c}{$34.90(15)$} \\
Distance $(d)$ & $\mathrm{pc}$ & \multicolumn{2}{c}{$553(32)$} \\
\hline
\end{tabular}

The effective temperature of the Sun was taken as $5780 \mathrm{~K}$, its bolometric absolute magnitude as 4.75 mag and its bolometric correction as $-0.07 \mathrm{mag}$.

Liégeois d'Évolution Stellaire (CLÉS, Scuflaire 2005). These computations were performed using the new solar abundances as reported by Asplund et al. (2005) and a standard mixing length description of convection with $\ell_{\mathrm{m}}=1.75$ times the local pressure scale height. The use of these models is appropriate for the components of CV Vel. Indeed, the $Z$-values we obtained for the primary and secondary are $Z=0.0119 \pm 0.0028$ and $Z=0.0160 \pm 0.0040$ for the case of an effective temperature of $18000 \mathrm{~K}$ and where we have taken the standard abundances of Grevesse \& Sauval (1998) for the elements not included in Table 6. The metallicities we obtain are thus consistent with those used in the model computations by De Cat et al. (2006). The tracks for $M=5.9,6.0,6.1 M_{\odot}$ are shown in Fig. 4 for two cases: no core overshoot and an overshoot of 0.2 times the local pressure scale height. We overplotted the position of CV Vel's components according to our results mentioned in Table 7. It can be seen that the agreement between our observational results and the evolutionary models is satisfactory. Recent results from asteroseismology have shown that a small core overshoot value is necessary to bring observed and identified oscillation frequencies in agreement with evolutionary models (Aerts et al. 2003; Pamyatnykh et al. 2004) in this part of the HR diagram. The systematic uncertainty for the temperatures (and thus luminosities) prevented us from constraining the overshoot parameters for the components of CV Vel. We derive an age of about 40 million years for the system. Clausen \& Grønbech (1977) reported an age of only about 30 million years, but they used much older models with somewhat different input physics (e.g., older opacity values).

\section{Study of the line-profile variability}

The components of CV Vel reside in the instability strip of the slowly pulsating B stars, not far away from the $\beta$ Cephei strip (Fig. 4). It is therefore worthwhile to search for short-period spectroscopic variations of the components. Percy \& Au-Young (2000) reported the possibility of a variation with an amplitude of $0.02 \mathrm{mag}$ and a period of 0.25 day in the Hipparcos photometry. However, they did not study CV Vel in detail. Clausen (private communication) was unable to find short-period variations in his extensive photometric data.

In Fig. 5a we plot the Mg II 4481 A lines of the components obtained at the phases outside of the eclipses. This line is the best one to study line-profile variability because it is one of the deeper ones and has the best $S / N$ level. We shifted all the lines to the common center of mass. The bold continuous line in Fig. 5b is the average profile. In Fig. 5c, the residuals of each line with respect to the average are shown. It is apparent from this figure that the primary shows line-profile variations.

We first estimated the rotational and thermal broadening from the average profiles shown in Fig. 5b. It is evident from this figure, as well as from Fig. 1, that the secondary rotates faster than the primary since both components essentially have the same thermal broadening. This is in contrast to Andersen's (1975) conclusion that the components have equal rotation velocity. We computed theoretical Mg II profiles for Gaussian intrinsic broadening as well as rotational broadening and find $v \sin i=19 \pm 1 \mathrm{~km} \mathrm{~s}^{-1}$ for the primary and $31 \pm 2 \mathrm{~km} \mathrm{~s}^{-1}$ for the secondary. Assuming the orbital and rotational axes to be 


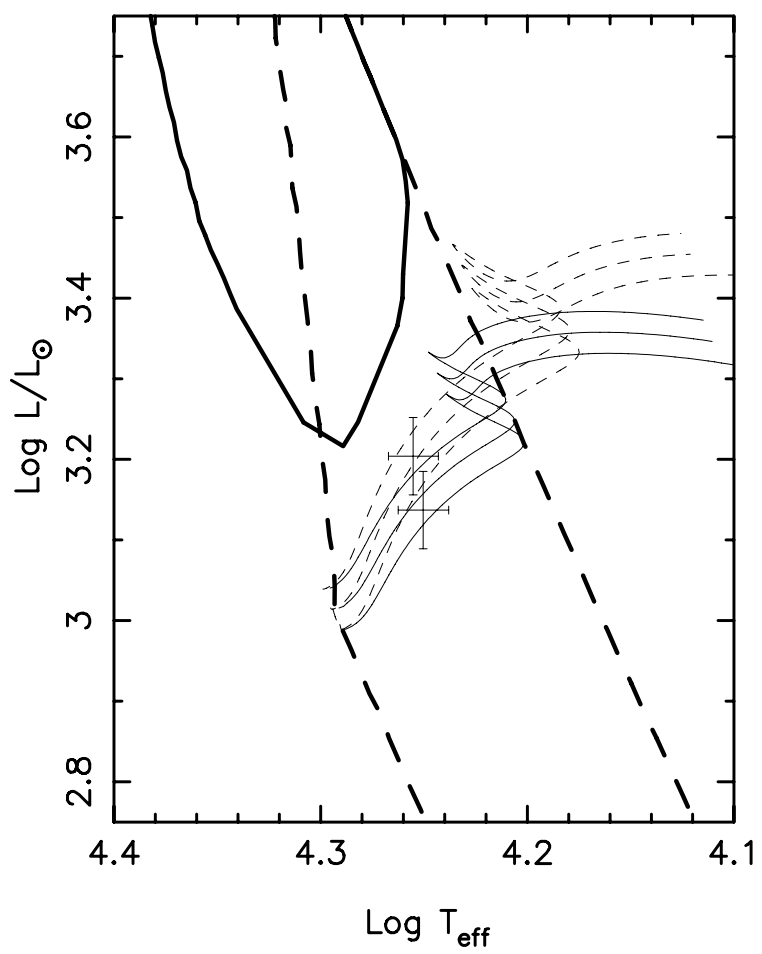

Fig. 4. The components of CV Vel in the HR diagram with error bars. The upper point represents the primary with $M=6.07 M_{\odot}$ and the lower point indicates the secondary with $M=5.97 M_{\odot}$ (see Table 7). The full thick line indicates the $\beta$ Cephei instability strip, the dashed thick line is the instability strip of the slowly pulsating B stars. Both strips were computed with the non-adiabatic oscillation code MAD (Dupret 2001) assuming that no core overshoot occurs. Evolutionary tracks computed with CLÉS are shown for $M=5.9,6.0,6.1 M_{\odot}$, and for zero core overshoot (thin full lines) as well as a core overshoot of 0.2 (thin dashed lines).

aligned, and using the radius estimates from Table 6 , we find a rotation period of $11.0( \pm 0.6)$ days for the primary and of 6.4 $( \pm 0.4)$ days for the secondary. It is noteworthy that the primary rotates subsynchronously, while the secondary's rotation period is compatible with the orbital period within the errors. Tidal theory (e.g., Zahn 1975, 1977) predicts the occurrence of synchronisation before circularisation, and both to happen on time scales shorter than about half the main-sequence duration, depending on the birth values of the eccentricity and orbital period. Deviations from synchronisation in circularised binaries have been reported before for close binaries with a B-type component and a similar orbital period, e.g. in the binary $\eta$ Ori with a pulsating component (De Mey et al. 1996).

We tried to quantify the line-profile variability of both components by computing the line moment variations in the definition of Aerts et al. (1992) for the 23 spectra shown in Fig. 5a. These line diagnostics stand for the centroid variation $(\langle v\rangle)$, the variation in the line width $\left(\left\langle v^{2}\right\rangle\right)$ and the variation in the line skewness $\left(\left\langle v^{3}\right\rangle\right)$. They are specifically powerful to unravel lowdegree $(\ell \leq 4)$ non-radial oscillation modes. We performed a frequency search with the Scargle method (Scargle 1982) on $\langle v\rangle$ in the range $[0.0,3.4] \mathrm{c} \mathrm{d}^{-1}$, where the upper limit of this interval corresponds to the Nyquist frequency. As a result, our dataset is not suitable to detect $\mathrm{p}$-mode oscillations in B2.5V stars because those have frequencies of typically 4 to $8 \mathrm{c} \mathrm{d}^{-1}$ for slow rotators (e.g. Aerts \& De Cat 2003). We should be able to find a signature of g-modes with amplitudes of a few $\mathrm{km} \mathrm{s}^{-1}$, as in slowly pulsating B stars (e.g. De Cat \& Aerts 2002).
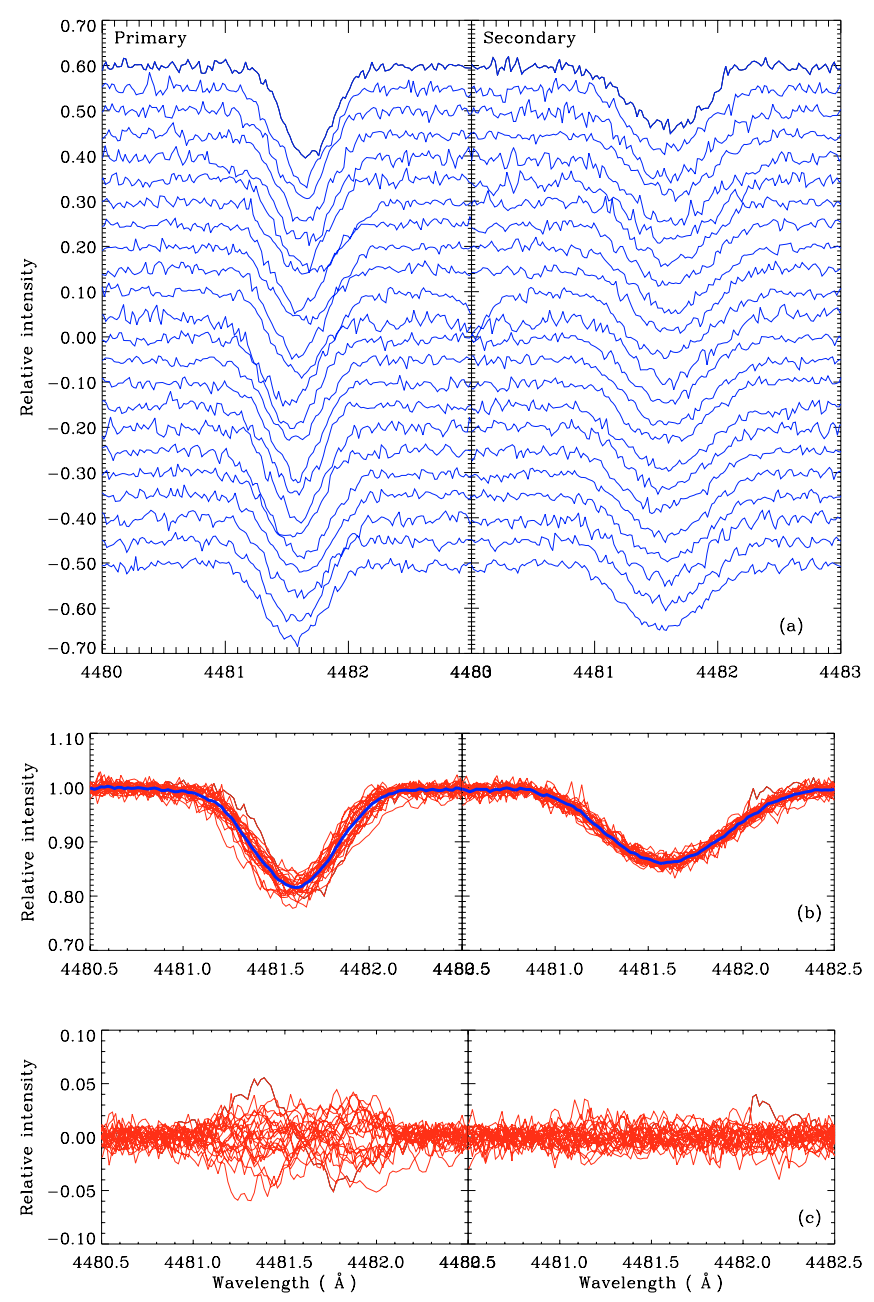

Fig. 5. a) Normalized Mg II ( $\lambda 4481$ ) line profiles of the primary (left panel) and the secondary (right panel). Phase runs from the top to the bottom. b) Line profiles superimposed. The full thick line is the average profile. c) Residual profiles with respect to the average.

The highest frequency peak in the Scargle periodogram of $\langle v\rangle$ for the primary occurs at $f=0.166 \pm 0.009 \mathrm{c} \mathrm{d}^{-1}$, with an amplitude of $2.2 \pm 0.4 \mathrm{~km} \mathrm{~s}^{-1}$. This frequency has a variance reduction of $54 \%$, which implies a decrease in the standard deviation from 2.3 to $1.5 \mathrm{~km} \mathrm{~s}^{-1}$. It reaches a level of $3.8 S / N$, where the $S / N$ level was computed from an oversampled Scargle periodogram of the residuals over the entire interval $[0.0,3.4] \mathrm{cd}^{-1}$. We recover the same frequency $f$ in $\left\langle v^{3}\right\rangle$. However, we do not find any periodic phenomenon in $\left\langle v^{2}\right\rangle$. A pulsation mode would imply the occurrence of $f$ and/or $2 f$ in $\left\langle v^{2}\right\rangle$ as well (Aerts et al. 1992, De Cat et al. 2005). It may be that our dataset is too limited in quality to detect a frequency in $\left\langle v^{2}\right\rangle$ given that the even moments are always noisier than the odd ones (Aerts et al. 1992).

For the secondary, we find $f^{\prime}=1.168 \pm 0.009 \mathrm{~cd}^{-1}$. This is a one-day alias of $f$. Forcing $f$ leads to an amplitude of $1.8 \pm 0.4 \mathrm{~km} \mathrm{~s}^{-1}$ (at level $4.1 S / N$ ) and a variance reduction of $51 \%$, bringing the standard deviation from 1.5 to $0.9 \mathrm{~km} \mathrm{~s}^{-1}$. This time, we cannot recover any frequencies in $\left\langle v^{2}\right\rangle$ or $\left\langle v^{3}\right\rangle$. We show in Fig. 6 the centroid velocity $\langle v\rangle$ for both the primary and secondary, folded with the frequency $f$, as well as their leastsquares fit. We find that the primary's $\langle v\rangle$ lags behind by a quarter of a phase compared to the one of the secondary.

We conclude that we have found evidence of periodic lineprofile variability in the primary and secondary of CV Vel with 


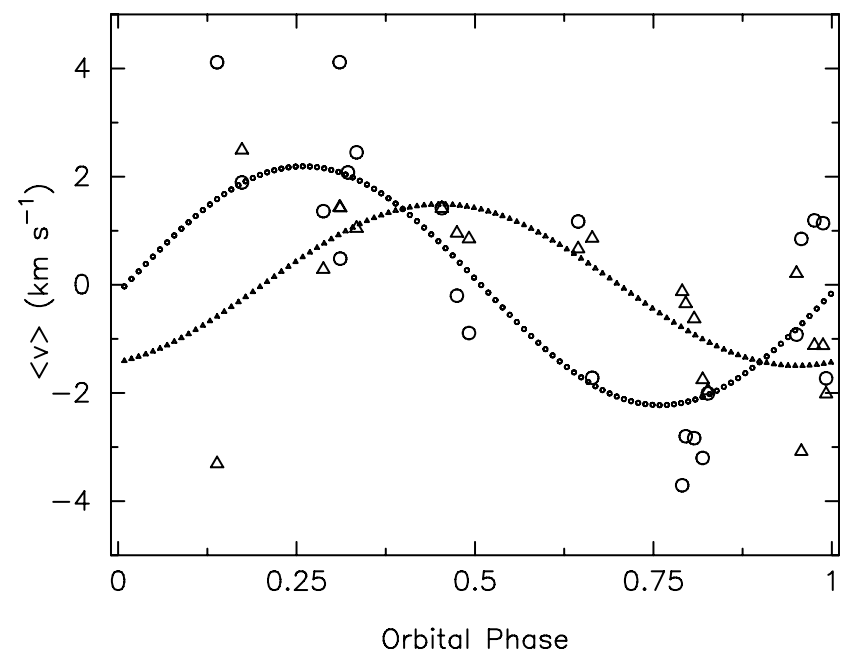

Fig. 6. The first moment variation as a function of orbital phase for the primary $(\circ)$ and secondary $(\triangle)$. The full lines with corresponding symbols are a least-squares sine fit.

a period between 5.7 and 6.4 days. This is almost equal to the orbital period. Moreover, the quoted frequency error is a formal $1 \sigma$ least-squares error, and is an underestimation of the true error given that we covered less than two full cycles with unblended $\mathrm{Mg}$ II lines. It therefore seems that line-profile variability occurs with a period very close or equal to the orbital one. This is potentially interesting as it could point towards the excitation of a tidally induced g-mode oscillation in the components. However, given the phase relation we obtained, and the absence of any periodic signature in $\left\langle v^{2}\right\rangle$, it is more likely that a reflection and/or variable limb-darkening effect lies at the origin of the detected variability. We need a more extensive data set to firmly establish the correct interpretation of CV Vel's line-profile variability.

\section{Summary}

From combined existing light and old and new radial-velocity curves, we have obtained a full solution of the double-lined eclipsing binary CV Vel. The system's center-of-mass velocity $\left(V_{0}\right)$ obtained by Feast (1954), Andersen (1975) and in this study are 27.9(1.1), 24.3(5), and 21.7(2) $\mathrm{km} \mathrm{s}^{-1}$, respectively. These three different and decreasing values may indicate the presence of a distant third body orbiting the binary system. Forthcoming observations of the system are necessary to evaluate this hypothesis. We checked but did not find a significant orbital period change.

We have corrected for the offset in $V_{0}$ in Andersen's (1975) and our data and merged these two high-quality radial-velocity curves to improve our knowledge of the fundamental parameters of the components. We complemented the spectroscopic data with high-quality 4-colour (uvby) lightcurves obtained by Clausen \& Grønbech (1977). These photometric and radialvelocity data were then solved simultaneously. The eclipsing and detached properties of the system have led to reliable orbital and physical parameters in agreement with the values obtained earlier by Clausen \& Grønbech (1977).

We presented for the first time an abundance analysis for the system and found results in agreement with those for B stars in the solar neighbourhood for both components. This analysis led to a systematic uncertainty of some $500 \mathrm{~K}$ for the effective temperatures of the components. Thus, these two parameters of this seemingly well-known system remain uncertain despite our

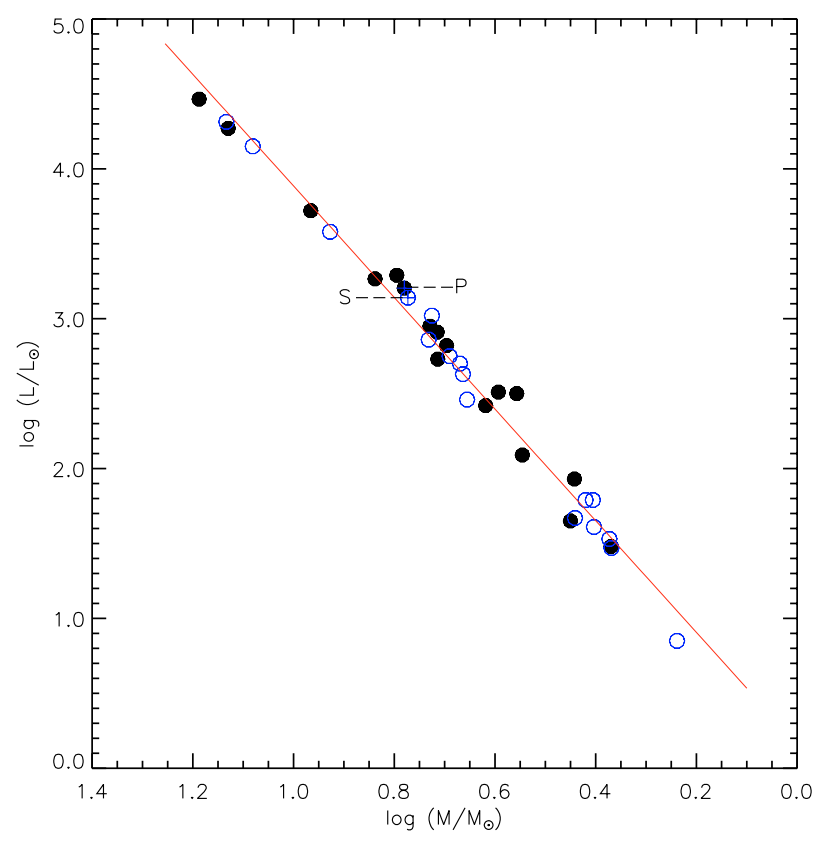

Fig. 7. Mass-Luminosity relation for well-known B-type eclipsing binaries. The filled and open circles represent the primary and the secondary components respectively. The components of CV Vel are indicated as $\mathrm{P}$ and $\mathrm{S}$.

detailed analysis based on échelle spectroscopy. It is not uncommon to find systematic uncertainties and deviations between photometrically and spectroscopically derived effective temperatures of B-type stars (e.g. De Ridder et al. 2004, for a discussion), and even to have uncertainties of the order of $500 \mathrm{~K}$ for such stars among methods based on the same data (e.g. Smalley \& Dworetsky 1995; Morel et al. 2006; Kaiser 2006). Previous works on CV Vel did not include a spectroscopic temperature estimate and were thus not able to estimate the systematic uncertainty of the component's temperatures.

Finally, we discovered line-profile variability in both components. The variability is most prominent in the primary. From the characteristics of the line profile moments, we tentatively interpreted this variability as due to an extrinsic orbital effect rather than an intrinsic oscillation. Any final interpretation of the detected line-profile variability requires a much more extensive dataset, however.

Our results show that $\mathrm{CV}$ Vel consists of young stars that are about half-way in their central hydrogen burning phase. Though the orbit is circular, the components clearly have different rotation speeds. We thus conclude that the tidal forces have had insufficient time to bring the system to a circular orbit with corotating components, i.e. the circularisation process is completed but not the synchronisation one.

Observational tests of stellar evolution models have been performed by many authors (Pols et al. 1997; Young et al. 2001; Lastennet \& Valls-Gabaus 2002; Young \& Arnett 2005, and references therein). To determine the masses and radii of stars without relying on models, one should use detached, eclipsing double-lined binaries and compute a simultaneous solution to their radial-velocity and multi-colour curves. Unfortunately, accurate physical parameters are only available for very few systems with early-type components. Andersen (1991) compiled a list of stars whose physical parameters are well defined, with relative mass and radius uncertainties less than $2 \%$. We collected all the well-known detached eclipsing binaries with main-sequence B-type components published since then (Table 8) and added 
Table 8. Observed parameters for B-type main-sequence double-lined eclipsing binary stars.

\begin{tabular}{llllllllllll}
\hline \hline Star & Sp.T & $P$ & $M_{1}$ & $M_{2}$ & $R_{1}$ & $R_{2}$ & $\log T_{e 1}$ & $\log T_{e 2}$ & $\log L_{1}$ & $\log L_{2}$ & Ref. \\
\hline AH Cep & B0.5Vn+B0.5Vn & 1.78 & 15.4 & 13.6 & 6.38 & 5.86 & 4.476 & 4.456 & 4.465 & 4.314 & 1 \\
CW Cep & B0.5V+B0.5V & 2.73 & 13.484 & 12.05 & 5.685 & 5.177 & 4.452 & 4.442 & 4.27 & 4.15 & 2 \\
QX Car & B2V+B2V & 4.48 & 9.245 & 8.46 & 4.289 & 4.051 & 4.377 & 4.354 & 3.72 & 3.58 & 2 \\
V497 Cep & B3V+B3V & 1.20 & 6.89 & 5.39 & 3.69 & 2.92 & 4.290 & 4.249 & 3.265 & 2.860 & 3 \\
V539 Ara & B3V+B4V & 3.17 & 6.239 & 5.313 & 4.432 & 3.734 & 4.26 & 4.23 & 3.29 & 3.02 & 2 \\
CV Vel & B2.5V+B2.5V & 6.89 & 6.066 & 5.972 & 4.126 & 3.908 & 4.255 & 4.250 & 3.20 & 3.14 & 4 \\
AG Per & B4V+B5V & 2.03 & 5.36 & 4.9 & 2.99 & 4.297 & 4.260 & 4.241 & 2.95 & 2.75 & 5 \\
U Oph & B5V+B5V & 1.68 & 5.186 & 4.672 & 3.438 & 3.005 & 4.22 & 4.199 & 2.91 & 2.7 & 2 \\
DI Her & B4V+B5V & 10.55 & 5.173 & 4.523 & 2.68 & 2.477 & 4.23 & 4.179 & 2.73 & 2.46 & 2 \\
V760 Sco & B4V+B4V & 1.73 & 4.968 & 4.609 & 3.013 & 2.64 & 4.228 & 4.21 & 2.82 & 2.63 & 2 \\
GG Lup & B7V+B9V & 1.85 & 4.155 & 2.532 & 2.411 & 1.753 & 4.176 & 4.041 & 2.42 & 1.61 & 2 \\
C Phe & B6V+B8V & 1.67 & 3.92 & 2.545 & 2.851 & 1.853 & 4.163 & 4.076 & 2.51 & 1.79 & 2 \\
X Hya & B8V+B8V & 2.27 & 3.605 & 2.631 & 4.384 & 2.165 & 4.066 & 4.041 & 2.5 & 1.79 & 2 \\
IQ Per & B8V+B6V & 1.74 & 3.513 & 1.732 & 2.446 & 1.503 & 4.09 & 3.885 & 2.09 & 0.85 & 2 \\
PV Cas & B9.5V+B9.5V & 1.75 & 2.82 & 2.761 & 2.243 & 2.287 & 4.000 & 4.000 & 1.65 & 1.67 & 2 \\
V451 Oph & B9V+A0V & 2.20 & 2.769 & 2.36 & 2.64 & 2.03 & 4.033 & 3.991 & 1.93 & 1.53 & 6 \\
GG Ori & B9.5V+B9.5V & 6.63 & 2.342 & 2.338 & 1.852 & 1.830 & 3.9978 & 3.9978 & 1.480 & 1.470 & 7 \\
\hline
\end{tabular}

References for Table 7: 1 Holmgren et al. (1990); 2 Andersen (1991) and references therein ; 3 Yakut et al. (2003); 4 this study; 5 Gimenez \& Clausen (1994); 6 Clausen et al. (1986); 7 Torres et al. (2000).

our new estimates of CV Vel. While we obtain a mass estimate with a relative precision of $1.2 \%$, the systematic uncertainty for the effective temperature that we derived from our high-quality spectra implies an uncertainty of some $12 \%$ on the luminosities of the components. Nevertheless, the values we obtained for CV Vel are fully compatible with evolutionary models and with those of similar systems whose parameters are known with better precision.

The stars given in Table 8 are drawn in a mass-luminosity plot in Fig. 7. From Table 8 we find the following update of the mass-luminosity relation for the main-sequence B-type stars:

$\log \left(L / L_{\odot}\right)=3.724(80) \log \left(M / M_{\odot}\right)+0.162(57)$.

Previously, Hilditch \& Bell (1987) presented a similar relation for $\mathrm{O}$ and $\mathrm{B}$ systems. The rms error in $\log (L)$ they found was 0.17 whereas it is only 0.11 for our Eq. (2) because we consider only binaries with B star components here. This equation is useful to predict masses from luminosities, or vice versa, for main-sequence B stars.

Acknowledgements. The authors are very much indebted to Prof. J. V. Clausen for sharing his photometric data of CV Vel with us, for encouraging us to study $\mathrm{CV}$ Vel spectroscopically and to perform an abundance analysis from our new data. Dr. B. Vandenbussche is acknowledged for performing the observations, Dr. M. Briquet for the help in the data preparation and Dr. P. De Cat for putting his grid of seismic models at our disposal. K.Y. warmly thanks Drs. S. Hony, M. Reyniers, H. Van Winckel and B. Kalomeni for their support. The authors are supported by the Research Council of the University of Leuven under grant GOA/2003/04 and a DB fellowship. We are very grateful to the anonymous referee for numerous comments and helpful remarks.

\section{References}

Aerts, C., De Pauw, M., \& Waelkens, C. 1992, A\&A, 266, 294

Aerts C., Thoul A., Daszyńska J., et al. 2003, Sci, 300, 1926

Aerts, C., \& De Cat, P. 2003, Space Sci. Rev., 105, 453

Andersen, J. 1975, A\&A, 44, 355

Andersen, J. 1991, A\&ARv, 3, 91

Asplund, M., Grevesse, N., \& Sauval, A. J. 2005, ASP Conf. Ser., 336, 25

Balona, L. A. 1994, MNRAS, 268, 119

Baranne, A., Queloz, D., Mayor, M., et al. 1996, A\&AS, 119, 373

Briquet, M., \& Morel, T. 2007, CoAst, in press

Clausen, J. V., \& Grønbech, B. 1977, A\&A, 58, 131
Clausen, J. V., Gimenez, A., \& Scarfe, C. 1986, A\&A, 167, 287

Daflon, S., \& Cunha, K. 2004, ApJ, 617, 1115

De Cat, P., \& Aerts, C. 2002, A\&A, 393, 965

De Cat, P., Telting, J., Aerts, C., \& Mathias, P. 2000, A\&A, 359, 539

De Cat, P., Briquet, M., Daszynska-Daszkiewicz, J., et al. 2005, A\&A, 432, 1013

De Cat, P., Briquet, M., Aerts, C., et al. 2007, A\&A, 463, 243

De Mey, K., Aerts, C., Waelkens, C., \& Van Winckel, H. 1996, A\&A, 310, 164

De Ridder, J., Telting, J. H., Balona, L. A., et al. 2004, MNRAS, 351, 324

Diaz-Cordoves, J., \& Gimenez, A. 1992, A\&A, 259, 227

Dupret, M.-A. 2001, A\&A, 366, 166

Feast, M. W. 1954, MNRAS, 114, 246

Gaposchkin, S. 1955, MNRAS, 115, 391

Gimenez, A., \& Clausen, J. V. 1994, A\&A, 291, 795

Grevesse, N., \& Sauval, J. 1998, SSRv, 85, 161

Hilditch, R. W., \& Bell, S. A. 1987, MNRAS, 229, 529

Holmgren, D. E., Hill, G., \& Fisher, W. 1990, A\&A, 236, 409

Hubscher, J. 2005, Informational Bulletin on Variable Stars, 5643

Kaiser, A. 2006, ASPC, 349, 257

Kurucz, R. L. 1993, ATLAS9 Stellar Atmosphere Programs and $2 \mathrm{~km} \mathrm{~s}^{-1}$ grid. Kurucz CD-ROM, Cambridge, Mass.: Smithsonian Astrophysical Observatory, 13

Lastennet, E., Valls-Gabaud, D., 2002, A\&A, 396, 551

Leung, K.-C., Zhai, D., \& Zhang, Y. 1985, AJ, 90, 515

Mathias, C., Aerts, C., De Pauw, M., Gillet, D., \& Waelkens, C. 1994, A\&A, 283,813

Mathias, P., \& Gillet, D. 1993 A\&A, 278, 511

Morel, T., Butler, K., Aerts, C., Neiner, C., \& Briquet, M. 2006, A\&A, 457, 651 Pamyatnykh A. A., Handler G., \& Dziembowski W. A. 2004, MNRAS, 350, 1022

Percy, J. R. \& Au-Yong, K., 2000, Informational Bulletin on Variable Stars, 4825 Pols, O. R., Tout, C. A., Schroder, K.-P., Eggleton, P. P., \& Manners, J. 1997, MNRAS, 289, 869

Savage, B. D., Massa, D., Meade, M., \& Wesselius, P. R. 1985, ApJS, 59, 397

Scargle J. D. 1981, ApJS, 45, 1

Scuflaire, R. 2005, CLÉS User Manual, Version 18, Liège University, Belgium

Smalley, B., \& Dworetsky, M. M. 1995, A\&A, 293, 446

Struve, O., \& Zebergs, V. 1960, ApJ, 130, 87

Torres, G., Lacy, C. H. Sandberg, C., Antonio, S., \& Jeffrey A. 2000, AJ, 120, 3226

Van Hoof, A. 1975, PASP, 69, 308

van Houten, C. J. 1950, Ann. Sterrew. Leiden, 20, 223

Wilson, R. E., \& Devinney, E. J. 1971, ApJ, 166, 605

Wilson R. E. 1994, PASP, 106, 921

Yakut, K., Tarasov, A. E., İbanoglu, C., et al. 2003, A\&A, 405, 1087

Young, P. A., Mamajek, E. E., Arnett, D., \& Liebert, J. 2001, ApJ, 556, 230

Young, P. A., \& Arnett, D. 2005, ApJ, 618, 908

Zahn, J.-P. 1975, A\&A, 41, 329

Zahn, J.-P. 1977, A\&A, 57, 383 